\title{
SYMMETRIC HOMOGENEOUS DIOPHANTINE EQUATIONS OF ODD DEGREE
}

\author{
M. A. REYNYA
}

\begin{abstract}
We find a parametric solution of an arbitrary symmetric homogeneous diophantine equation of 5 th degree in 6 variables using two primitive solutions. We then generalize this approach to symmetric forms of any odd degree by proving the following results.

(1) Every symmetric form of odd degree $n \geq 5$ in $6 \cdot 2^{n-5}$ variables has a rational parametric solution depending on $2 n-8$ parameters.

(2) Let $F\left(x_{1}, \ldots, x_{N}\right)$ be a symmetric form of odd degree $n \geq 5$ in $N=6 \cdot 2^{n-4}$ variables, and let $q$ be any rational number. Then the equation $F\left(x_{i}\right)=q$ has a rational parametric solution depending on $2 n-6$ parameters.

The latter result can be viewed as a solution of a problem of Waring type for this class of forms.
\end{abstract}

\section{INTRODUCTION}

Given any form $F\left(x_{1}, \ldots, x_{N}\right)$ with rational coefficients, one can look for conditions under which the equation $F\left(x_{1}, \ldots, x_{N}\right)=0$ has a nonzero rational solution. It is known that if $N$ is big enough with respect to the degree of $F$ (which is assumed odd), such a solution always exists (see [Bi1], [Bi2]; more recent papers [W1], [W2] provide explicit estimates for $N$ ). In the present paper, we consider symmetric forms $F\left(x_{1}, \ldots, x_{N}\right)$, i.e. forms invariant under the natural action of the symmetric group $S_{N}$ on variables. For such a form the existence of a nonzero rational solution is guaranteed, and we are interested in getting parametric solutions and estimating the number of parameters. More precisely, for any given integers $n$ and $k$ we want to find an integer $N$ such that every symmetric form in $N$ variables of degree $2 n+1$ with rational coefficients has a parametric solution depending on $k$ parameters.

Our method, being essentially elementary, is inspired by a geometric approach proposed by Manin [M] for studying rational points on cubic hypersurfaces: given a few rational points on such a variety, one can use a composition law in order to produce new rational points. We implement a similar idea in the case of symmetric forms of odd degree: starting from two "primitive" rational points, we propose an algorithm producing lots of rational points.

To explain our method, we begin with the case of quintics in 6 variables (see Section 2). We prove that any such quintic has a parametric solution $\left(x_{1}, \ldots, x_{6}\right)$ such that $x_{1}+\cdots+x_{6}=0$ (Theorem 2.41). Note that to the best of our knowledge, such a result did not appear in 
the literature, even in the simplest case of diagonal quintics. Our method is significantly different both from the elementary approach in $[\mathrm{R}],[\mathrm{C}]$ and computer investigation of [LP], [LPS], [E]. It can be compared with geometric constructions in $[\mathrm{Br}]$ which led to some new insight on parametric solutions obtained in [SwD].

Next, in Section 3, we extend our method to symmetric forms of arbitrary odd degree. Our main result (Theorem 3.1) states that every symmetric form of odd degree $n \geq 5$ in $6 \cdot 2^{n-5}$ variables has a rational parametric solution depending on $2 n-8$ parameters. As an application, we obtain a solution of the following version of Waring's problem (Theorem 3.2):

Let $F\left(x_{1}, \ldots, x_{N}\right)$ be a symmetric form of odd degree $n \geq 5$ in $N=6 \cdot 2^{n-4}$ variables, and let $q$ be any rational number. Then the equation $F\left(x_{i}\right)=q$ has a rational parametric solution depending on $2 n-6$ parameters.

\section{Symmetric QUintics in 6 VARIABLES}

We begin with a simple general observation:

Lemma 2.1. Every symmetric form of degree $2 n+1$ in $2 N \geq 6$ variables has a zero of the form

$$
\left(a_{1},-a_{1}, \ldots, a_{N-1},-a_{N-1}, 1,-1\right)
$$

with $a_{i} \neq a_{j} \neq 1$.

Proof. By a well-known algebraic theorem, every symmetric polynomial $P \in k\left[x_{1}, \ldots, x_{N}\right]^{S_{N}}$ can be represented as a polynomial in elementary symmetric polynomials $e_{1}\left(x_{1}, \ldots, x_{N}\right), \ldots$, $e_{N}\left(x_{1}, \ldots, x_{N}\right)$. In particular, every symmetric form can be represented as

$$
F=\sum_{i_{1}+\cdots+i_{k}=2 n+1} \alpha_{i_{1}, \ldots, i_{k}} e_{i_{1}} \ldots e_{i_{k}}
$$

where $e_{i_{m}}\left(x_{1}, \ldots, x_{N}\right)$ stands for the elementary symmetric polynomial of degree $i_{m}$. Each summand contains a factor of odd degree, therefore they all vanish at any point of the form (1).

Definition 2.2. We call any point of the form (1), as well as any its image with respect to the action of the symmetric group, a primitive solution of $F=0$.

First consider the case of diagonal quintics. 
Proposition 2.3. The equation $x_{1}^{5}+x_{2}^{5}+x_{3}^{5}+x_{4}^{5}+x_{5}^{5}+x_{6}^{5}=0$ has a parametric solution, where $x_{i}$ are polynomials in two parameters $a$ and $b$, and $x_{1}+\cdots+x_{6}=0$.

Proof. This equation has primitive solutions $(a,-a, b,-b, 1,-1)$ and $(-1, c,-c, d,-d, 1)$. Let us look for a new solution in the form

$$
(a \cdot t-1)^{5}+(-a \cdot t+c)^{5}+(b \cdot t-c)^{5}+(-b \cdot t+d)^{5}+(1 \cdot t-d)^{5}+(-1 \cdot t+1)^{5}=0 .
$$

This gives an equation in $t$. For every $t$ we have $x_{1}+\cdots+x_{6}=0$. When $t$ runs over the projective line, this equation has 5 roots: $t_{1}=0, t_{2}=\infty$, and 3 additional roots: $t_{3}, t_{4}, t_{5}$.

The main idea is as follows: to get these 3 remaining roots $t_{3}, t_{4}, t_{5}$, we have an equation in $t$ of degree 3 obtained from (2):

$$
A \cdot t^{3}+B \cdot t^{2}+C \cdot t+D=0
$$

where $A, B, C, D$ are functions in $a, b, c, d$; let us require $A=0, B=0$. Solving this system of two equations, we get $c, d$ as functions in $a$ and $b$. Then $t=-D / C, D$ and $C$ are also functions in $a$ and $b$. We have

$$
\begin{aligned}
& A=-a^{4}+a^{4} \cdot c-b^{4} \cdot c+b^{4} \cdot d-d+1, \\
& B=a^{3}-a^{3} \cdot c^{2}+b^{3} \cdot c^{2}-b^{3} \cdot d^{2}+d^{2}-1 .
\end{aligned}
$$

If $A=0$ then $c=\left(d \cdot\left(1-b^{4}\right)+a^{4}-1\right) /\left(a^{4}-b^{4}\right)$.

If $B=0$ then $c^{2}=\left(d^{2} \cdot\left(b^{3}-1\right)+1-a^{3}\right) /\left(b^{3}-a^{3}\right)$.

We have a system of two equations, where $c$ and $d$ are unknowns. It is easy to transform this system into one equation of degree 2 in $d$; then this equation evidently has a solution $d=1$, because $c=d=1$ is a solution of this system, so the second root is $d=f(a, b)$.

This method can be extended to more complicated cases.

Theorem 2.4. Every symmetric quintic equation $F\left(x_{1}, \ldots, x_{6}\right)=0$ has a parametric solution where $x_{i}$ are polynomials in two parameters $a$ and $b$, and $x_{1}+\cdots+x_{6}=0$.

Proof. Under the assumption $x_{1}+\cdots+x_{6}=0$, every quintic is representable as

$$
A_{1} \cdot\left(x_{1}^{5}+\cdots+x_{6}^{5}\right)+A_{2} \cdot\left(x_{1}^{3}+\cdots+x_{6}^{3}\right) \cdot\left(x_{1}^{2}+\cdots+x_{6}^{2}\right) .
$$

Indeed, any symmetric quintic can be represented as a polynomial in diagonal forms:

$$
\begin{aligned}
A_{1}\left(x_{1}^{5}+\cdots+x_{6}^{5}\right) & +A_{2}\left(x_{1}^{3}+\cdots+x_{6}^{3}\right) \cdot\left(x_{1}^{2}+\cdots+x_{6}^{2}\right)+A_{3}\left(x_{1}^{4}+\cdots+x_{6}^{4}\right) \cdot\left(x_{1}+\cdots+x_{6}\right) \\
& +A_{4}\left(x_{1}^{3}+\cdots+x_{6}^{3}\right) \cdot\left(x_{1}+\cdots+x_{6}\right)^{2}+A_{5}\left(x_{1}+\cdots+x_{6}\right) \cdot\left(x_{1}^{2}+\cdots+x_{6}^{2}\right)^{2} \\
& +A_{6}\left(x_{1}+\cdots+x_{6}\right)^{3} \cdot\left(x_{1}^{2}+\cdots+x_{6}^{2}\right)+A_{7}\left(x_{1}+\cdots+x_{6}\right)^{5},
\end{aligned}
$$


and all terms, except for the first two ones, vanish. To find a parametric solution of the resulting quintic, we use exactly the same method as for the diagonal quintic in Proposition 2.3 .

This quintic has primitive solutions

$$
(a,-a, b,-b, 1,-1),(-1, c,-c, d,-d, 1) \text {. }
$$

Let us look for a new solution in the form:

$$
\begin{aligned}
& \quad A_{1} \cdot\left((a \cdot t-1)^{5}+(-a \cdot t+c)^{5}+(b \cdot t-c)^{5}+(-b \cdot t+d)^{5}+(1 \cdot t-d)^{5}+(-1 \cdot t+1)^{5}\right)+ \\
& A_{2}\left((a \cdot t-1)^{3}+(-a \cdot t+c)^{3}+(b \cdot t-c)^{3}+(-b \cdot t+d)^{3}+(1 \cdot t-d)^{3}+(-1 \cdot t+1)^{3}\right)((a \cdot t- \\
& \left.1)^{2}+(-a \cdot t+c)^{2}+(b \cdot t-c)^{2}+(-b \cdot t+d)^{2}+(1 \cdot t-d)^{2}+(-1 \cdot t+1)^{2}\right)=0 .
\end{aligned}
$$

This gives an equation in $t$. We use the same idea: this equation in $t$ has 5 roots: $0, \infty$, $t_{1}, t_{2}, t_{3}$. For $t_{1}, t_{2}, t_{3}$ we have an equation:

$$
R_{1} \cdot t^{3}+R_{2} \cdot t^{2}+R_{3} \cdot t+R_{4}=0
$$

where $R_{1}, R_{2}, R_{3}, R_{4}$ are functions in $a, b, c, d, A_{1}, A_{2}$. Now we require: $R_{1}=0, R_{2}=0$. This is a system of two equations with unknowns $c$ and $d$. The equation $R_{1}=0$ is linear in $c$ and $d$, and the equation $R_{2}=0$ is quadratic in $c$ and $d$. It is easy to see that if we transform this system to one equation of degree 2 in $d$, this equation evidently has the root $d_{1}=1$ corresponding to the primitive solution

$$
(a \cdot t-1,-a \cdot t+1, b \cdot t-1,-b \cdot t+1, t-1,-t+1) .
$$

The second root is $d_{2}=F(a, b)$. So the equation in $t$ reduces to the equation:

$$
K_{1}(a, b) \cdot t+K_{2}(a, b)=0
$$

from which we can express $t$ as a function in $a$ and $b$. We thus find a parametric solution for any symmetric diophantine equation of degree 5 in 6 variables: $x_{i}=f_{i}(a, b)$ for which we have $x_{1}+\cdots+x_{6}=0$.

\section{General Results}

In this section we generalize Theorem 2.4 to the case of a form of an arbitrary odd degree.

Theorem 3.1. Let $F$ be a symmetric form in $N$ variables of odd degree $n \geq 5$ with rational coefficients. If $N \geq 6 \cdot 2^{n-5}$ then the equation $F\left(x_{1}, \ldots, x_{N}\right)=0$ has a parametric solution where $x_{i}$ are polynomials in $s=2 n-8$ parameters, and $x_{1}+\cdots+x_{N}=0$. 
Proof. First consider the equation

$$
\begin{aligned}
\left(x_{1}^{5}+\cdots+x_{6}^{5}\right) & +\left(x_{1}^{4}-x_{2}^{4}+x_{3}^{4}-x_{4}^{4}+x_{5}^{4}-x_{6}^{4}\right) \cdot D_{1}\left(x_{i}\right) \\
& +\left(x_{1}^{3}+x_{2}^{3}+x_{3}^{3}+x_{4}^{3}+x_{5}^{3}+x_{6}^{3}\right) \cdot D_{2}\left(x_{i}\right) \\
& +\left(x_{1}^{2}-x_{2}^{2}+x_{3}^{2}-x_{4}^{2}+x_{5}^{2}-x_{6}^{2}\right) \cdot D_{3}\left(x_{i}\right)=0,
\end{aligned}
$$

where $D_{1}\left(x_{i}\right)$ is a polynomial in $x_{i}$ of degree at most $1, D_{2}\left(x_{i}\right)$ is a polynomial in $x_{i}$ of degree at most $2, D_{3}\left(x_{i}\right)$ is a polynomial in $x_{i}$ of degree at most 3 . It is easy to see that this equation has solutions:

$$
(a,-a, b,-b, 1,-1),(-1, c,-c, d,-d, 1) \text {. }
$$

Let us try to find a new solution by the same method:

$$
x_{1}=a \cdot t-1, x_{2}=-a \cdot t+c, x_{3}=b \cdot t-c, x_{4}=-b \cdot t+d, x_{5}=t-d, x_{6}=-t+1,
$$

so equation (4) in $t$ has roots $t=0, t=\infty$, and hence can be transformed to an equation of degree 3

$$
S_{1} \cdot t^{3}+S_{2} \cdot t^{2}+S_{3} \cdot t+S_{4}=0
$$

(here $S_{1}, S_{2}, S_{3}, S_{4}$ are functions in $a, b, c, d$ ).

Now, as above, we have two equations for $c$ and $d: S_{1}=0, S_{2}=0$. The equation $S_{1}=0$ is linear in $c$ and $d$, and $S_{2}=0$ is quadratic in $c$ and $d$. From the equation $S_{1}=0$ it follows that if $d=1$ then $c=1$, because for $c=1, d=1$, equation (4) becomes an identity. Therefore the equation $S_{2}=0$ must have the root $d_{2}=1, d_{1}=F(a, b)$, and equation (44) is transformed to $S_{3} \cdot t+S_{4}=0$. So we can set $t=-S_{4} / S_{3}$ and find a parametric solution of equation (4).

Now suppose that we have an arbitrary symmetric form $F$ of degree $n=2 k+1$, and the number of variables is $N=4 s$. For every quadruple of variables we use a transformation of the form

$$
z_{1}=x_{1}+c_{1}, z_{2}=-x_{2}+d_{1}, z_{3}=-x_{1}-d_{1}, z_{4}=x_{2}-c_{1} .
$$

We represent this form as follows:

$$
z_{1}^{2 n+1}+\cdots+z_{N}^{2 n+1}+A_{1}\left(z_{1}^{2 n-1}+\cdots+z_{N}^{2 n-1}\right) R_{1}\left(z_{i}\right)+\cdots+A_{k}\left(z_{1}+\cdots+z_{N}\right) R_{k}\left(z_{i}\right),
$$

where $R_{j}\left(z_{i}\right)$ are symmetric polynomials. Let us now look how this transformation works.

It is easy to see that we obtain a form of degree $2 n$ whose coefficients are functions in $c_{N}$, $d_{N}$, but we obtain a new symmetric construction of the form

$$
x_{1}^{2 n}-x_{2}^{2 n}+x_{3}^{2 n}-x_{4}^{2 n}+\ldots
$$


Under transformation (5), polynomials of this type go over to linear combinations of symmetric diagonal polynomials of odd degree and polynomials of the form (6) of even degree. The degree of these polynomials is smaller than $2 n+1$. Repeating this transformation several times, we obtain an equation of the form

$$
\begin{aligned}
\left(x_{1}^{5}+\cdots+x_{6}^{5}\right) & +\left(x_{1}^{4}-x_{2}^{4}+x_{3}^{4}-x_{4}^{4}+x_{5}^{4}-x_{6}^{4}\right) \cdot D_{1}\left(x_{i}\right) \\
& +\left(x_{1}^{3}+x_{2}^{3}+x_{3}^{3}+x_{4}^{3}+x_{5}^{3}+x_{6}^{3}\right) \cdot D_{2}\left(x_{i}\right) \\
& +\left(x_{1}^{2}-x_{2}^{2}+x_{3}^{2}-x_{4}^{2}+x_{5}^{2}-x_{6}^{2}\right) \cdot D_{3}\left(x_{i}\right)=0,
\end{aligned}
$$

but for this equation the existence of a solution depending on two parameters $a$ and $b$ is proved in Theorem 2.4. So if a symmetric form has $6 \cdot 2^{n-5}(n \geq 5$ is an odd number) variables, it has a parametric solution in $s=2 n-8$ parameters.

One can use the same approach as in Theorem 3.1 for finding parametric solutions of the following problem of Waring's type.

Theorem 3.2. Let $n \geq 5$ be an odd integer, $N=6 \cdot 2^{n-4}$, let $F$ be a symmetric form of degree $n$ with rational coefficients, and let $q$ be a fixed rational number. Then the equation $F\left(x_{1}, \ldots, x_{N}\right)=q$ has a parametric solution depending on $s=2 n-6$ parameters.

Proof. The proof follows, almost word for word, the arguments of Theorem 3.1, and we leave the details to a scrupulous reader.

Remark 3.3. One can pose the following problem: for given integers $n$ and $k$ to find an integer $N$ such that every symmetric form in $N$ variables of degree $2 n+1$ with rational coefficients has a parametric solution depending on $k$ parameters. Such a problem can be treated using the approach proposed in this paper. In particular, it is very easy to generalize the results of this paper from symmetric quintics in 6 variables to symmetric quintics in any even number of variables. One can use primitive solutions to construct, for every symmetric quintic in $2 P$ variables, a parametric solution depending on $P-1$ parameters. Using transformation (5), one can then generalize this result for every symmetric form of odd degree. Finally, for every given integers $n$ and $k$ one can find an integer $N$ such that every symmetric form in $N$ variables of degree $2 n+1$ with rational coefficients has a parametric solution depending on $s$ parameters with $s>k$. If in this solution we substitute arbitrary rational numbers instead of $s-k$ parameters, we obtain a desired parametric solution depending on $k$ parameters. 


\section{REFERENCES}

[Bi1] B. J. Birch, Homogeneous forms of odd degree in a large number of variables, Mathematika 4 (1957) 102-105.

[Bi2] B. J. Birch, Forms in many variables, Proc. Roy. Soc. Ser. A 265 (1961/1962) 245-263.

[Br] A. A. Bremner, Geometric approach to equal sums of fifth powers, J. Number Theory 13 (1981) $337-354$.

[C] A. Choudhry, On equal sums of fifth powers, Indian J. Pure Appl. Math. 28 (1997) 1443-1450.

[E] R. L. Ekl, New results in equal sums of like powers, Math. Comput. 67 (1998) 1309-1315.

[LP] L. J. Lander and T. R. Parkin, A counterexample to Euler's sum of powers conjecture, Math. Comput. 21 (1967) 101-103.

[LPS] L. J. Lander, T. R. Parkin, and J. L. Selfridge, A survey of equal sums of like powers, Math. Comput. 21 (1967) 446-459.

[M] Yu. I. Manin, Cubic Forms: Algebra, Geometry, Arithmetic, Nauka, Moscow, 1972; 2nd ed. of the English transl. North-Holland, Amsterdam, 1986.

[R] K. S. Rao, On sums of fifth powers, J. London Math. Soc. 9 (1934) 170-171.

[SwD] H. P. F. Swinnerton-Dyer, A solution of $A^{5}+B^{5}+C^{5}=D^{5}+E^{5}+F^{5}$, Proc. Cambridge. Phil. Soc. 48 (1952) 516-518.

[W1] T. D. Wooley, Forms in many variables, Analytic Number Theory (Kyoto, 1996), London Math. Soc. Lecture Note Ser., vol. 247, Cambridge Univ. Press, Cambridge, 1997, pp. 361-376.

[W2] T. D. Wooley, An explicit version of Birch's theorem, Acta Arith. 85 (1998) 79-96.

Ahva St. 15/14, Haifa, IsRael

E-mail address: misha_371@mail.ru 